\title{
Correlating local structure and sodium storage in hard carbon anodes: insights from pair distribution function analysis and solid-state NMR
}

\author{
Joshua M. Stratford, ${ }^{\dagger \#}$ Annette K. Kleppe, ॥ Dean S. Keeble,\| Philip A. Chater,\| \\ Seyyed Shayan Meysami, $\sim$ Christopher J. Wright, $\sim$ Jerry Barker, $\sim$ Maria-Magdalena Titirici, ${ }^{+}$ \\ Phoebe K. Allan, ${ }^{* \#}$ Clare P. Grey ${ }^{* \dagger}$ \\ $\dagger$ Department of Chemistry, University of Cambridge, Lensfield Road, Cambridge, CB2 1EW, U.K. \\ * School of Chemistry, University of Birmingham, Edgbaston, Birmingham, B15 2TT, U.K. \\ $\|$ Diamond Light Source Ltd., Harwell Science and Innovation Campus, Didcot, OX11 ODE, U.K. \\ $\sim$ Faradion Limited, The Innovation Centre, 217 Portobello, Sheffield, S1 4DP, U.K. \\ + Department of Chemical Engineering, Imperial College London, London, SW7 2AZ, UK.
}

* Corresponding authors:

Dr Phoebe K. Allan, email: p.allan@bham.ac.uk

Professor Clare P. Grey, email: cpg27@cam.ac.uk

\section{Supplementary Information}

1. Elemental analysis of pristine carbons

2. Total scattering data for the pristine carbons

3. Simulations of the reduced structure functions for equally spaced curved graphene fragments

4. Comparison of the operando NMR spectrum for each carbon at the end of the first discharge

5. Total scattering data for Carbon A with electrolyte

6. Small angle scattering data for Carbon B

7. $\mathrm{dQ} / \mathrm{dV}$ data for each carbon

8. Scale factors for operando PDF

9. Differential PDFs during the charge processes

10. Creating a curved graphene fragment

11. Differential PDF deconvolution method

12. Modelling the broad component of the differential PDF for the sloping process

13. Calculated PDFs for different sodium cluster diameters

14. References 


\section{ELEMENTAL ANALYSIS OF PRISTINE CARBONS}

Table S1 shows the composition of each of the carbons studied. In all cases, we see $\geq 90$ w.t. $\%$ carbon, for the higher temperature carbons, and for the commercially relevant samples (Carbons A and B) $\geq 97$ w.t.\% carbon is seen.

Table S1: The C, H, and N wt. \% for each of the carbons as determined by elemental analysis. The remainder is assumed to be oxygen.

\begin{tabular}{ccccc} 
Carbon & $\mathrm{C}$ & $\mathrm{H}$ & $\mathrm{N}$ & O (assumed) \\
\hline $900{ }^{\circ} \mathrm{C}$ & 90.2 & 0.4 & 0.0 & 9.6 \\
$1000^{\circ} \mathrm{C}$ & 94.2 & 0.5 & 0.3 & 5.0 \\
$1100^{\circ} \mathrm{C}$ & 97.1 & 0.4 & 0.0 & 2.5 \\
$1500^{\circ} \mathrm{C}$ & 100.0 & 0.0 & 0.0 & 0.0 \\
Carbon A & 97.2 & 0.0 & 0.0 & 2.8 \\
Carbon B & 99.5 & 0.0 & 0.0 & 0.5
\end{tabular}




\section{TOTAL SCATTERING DATA FOR THE PRISTINE CARBONS}

Ex situ PDF measurements were taken at beamline I15-1 (XPDF) at Diamond Light Source using $2.5 \mathrm{~mm}$ quartz capillaries. Measurements were taken using an x-ray beam of energy $76 \mathrm{keV}(\lambda=0.1631 \AA)$ and an amorphous silicon area detector (Perkin Elmer). The total exposure time per scan was $900 \mathrm{~s}$. A background measurement was taken using an empty capillary. A Si standard was used to determine the sample geometry and the sample-to-detector distance. The data were converted to a function of intensity vs. Q using Data Analysis WorkbeNch (DAWN). ${ }^{1}$ Standard corrections (background, Compton scattering, detector effects) were applied, and the data Fourier transformed to obtain G(r) using the software PDFGetX2. ${ }^{2}$

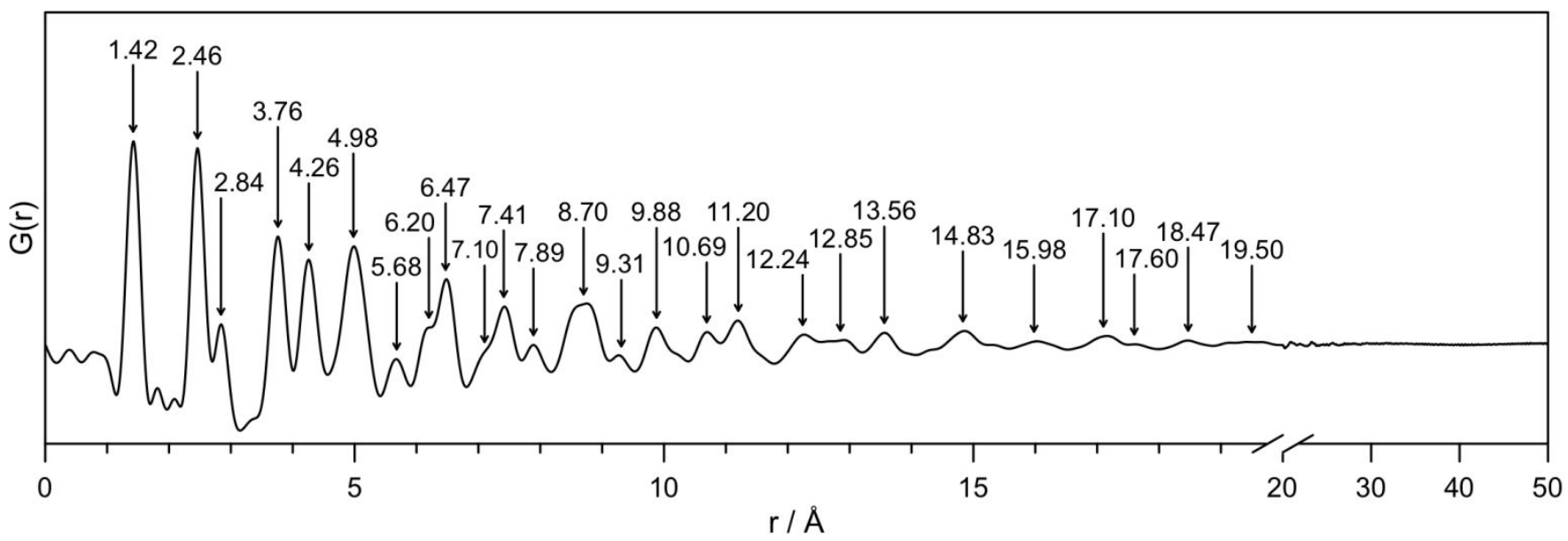

Figure S1: Experimental PDF data for a commercial hard carbon (Carbon A), significant peaks $r<20 \AA$ are indicated.
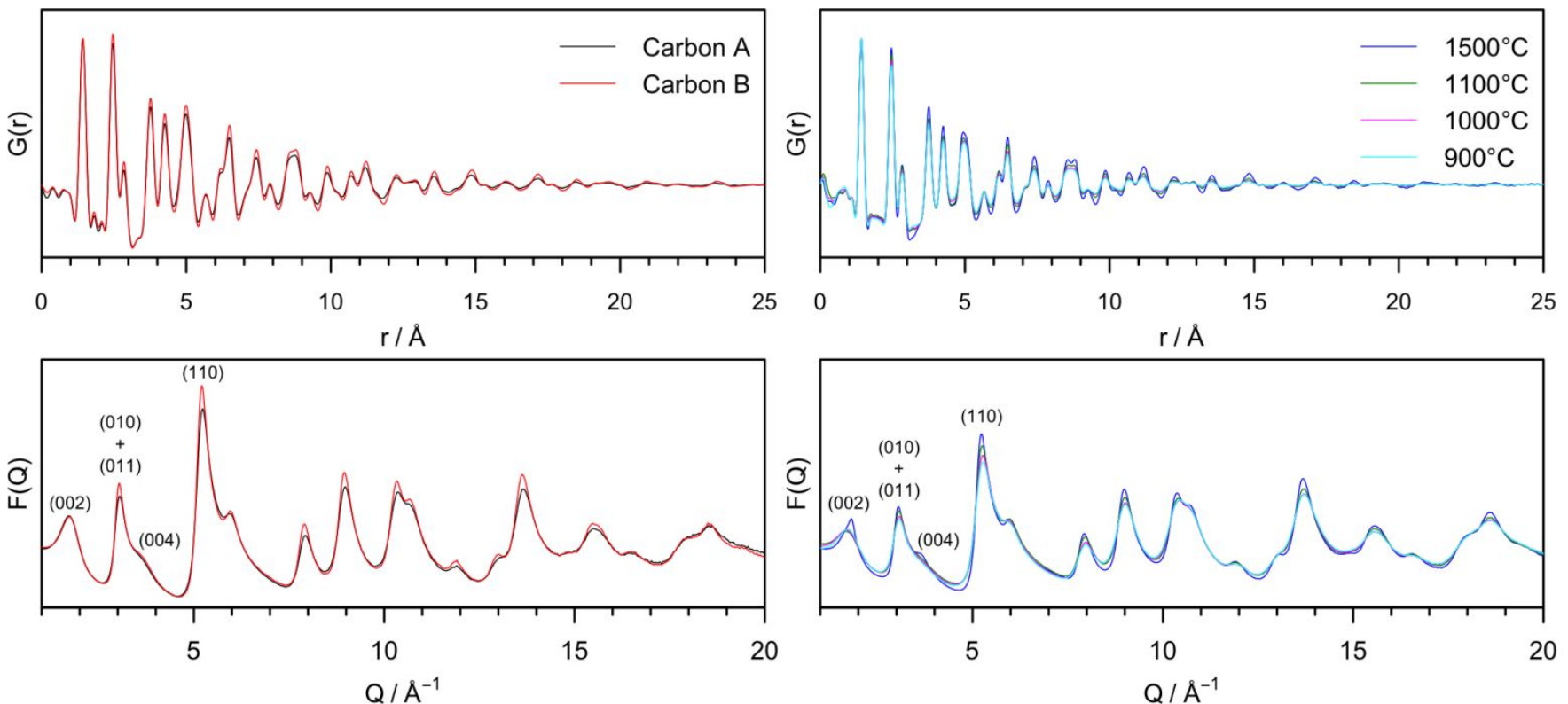

Figure S2: A comparison of the PDFs and reduced structure functions, $F(Q)$, for two commercially relevant carbons: Carbon A (black), and Carbon B (red), and four glucose-derived carbons with pyrolysis temperatures of $900{ }^{\circ} \mathrm{C}$ (cyan), $1000^{\circ} \mathrm{C}$ (magenta), $1100^{\circ} \mathrm{C}$ (green), and $1500^{\circ} \mathrm{C}$ (blue). $F(Q)$ peaks below $6 \AA^{-1}$ are indexed based on the graphite unit cell. 


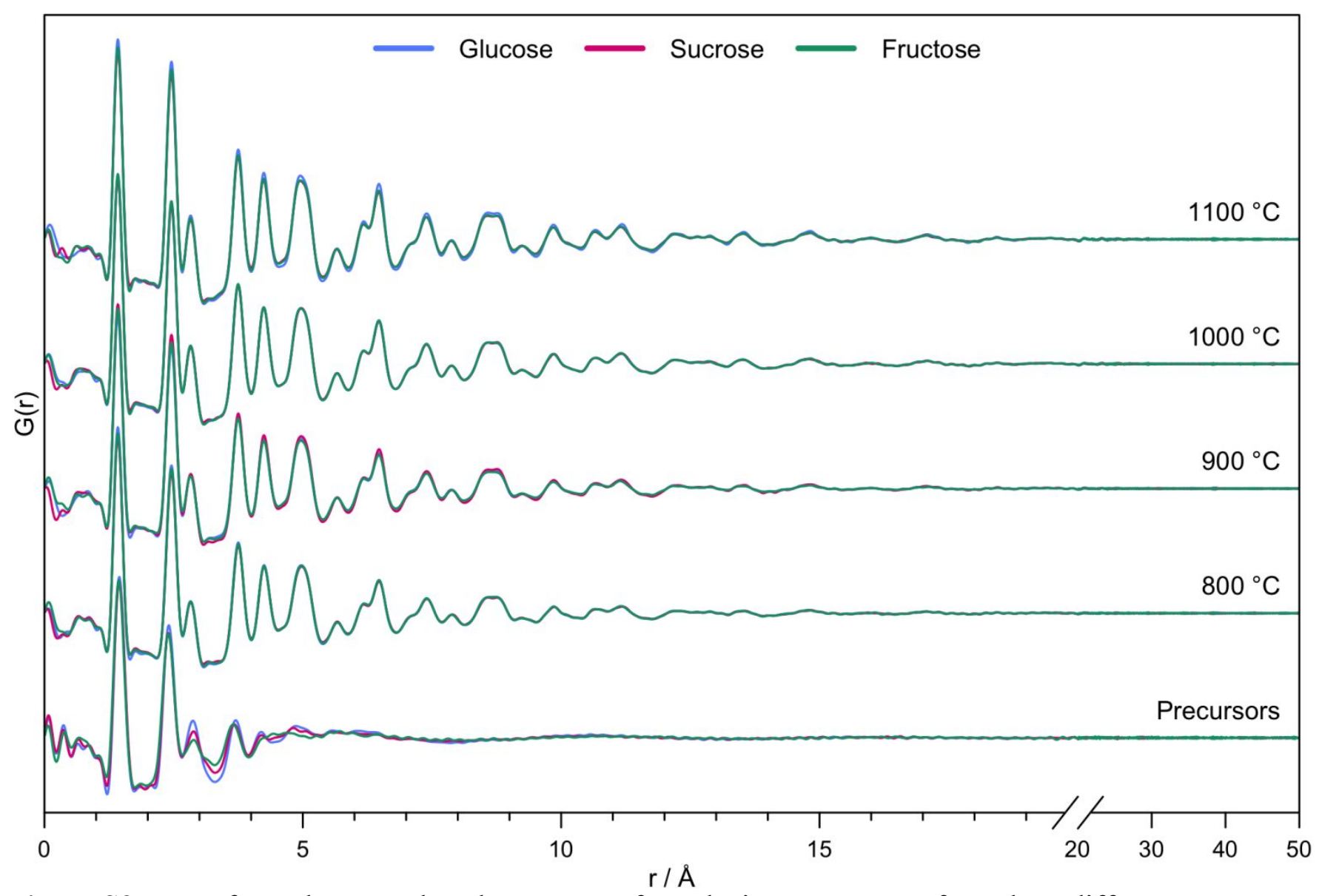

Figure S3: PDFs for carbons produced at a range of pyrolysis temperatures from three different precursors. Carbons produced using a glucose precursor are shown in blue, sucrose in pink and fructose in green. PDFs for the dehydrated precursors (caramels) are shown below. 


\section{SIMULATIONS OF THE REDUCED STRUCTURE FUNCTION FOR EQUALLY SPACED CURVED GRAPHENE FRAGMENTS}

A curved graphene fragment, sized $25 \times 25 \AA$, was created using the DiffPy-CMI complex modelling infrastructure software. ${ }^{3}$ Multiple fragments were stacked in an eclipsed configuration, with the interlayer distance being $3.7 \AA$. The total number of layers was varied between 1 and 10 , and the reduced structure function $F(Q)$ calculated from a Debye sum between 0 and $23 \AA$. The Qdamp parameter was set to 0.03 , and the delta1 parameter to 0.23 .

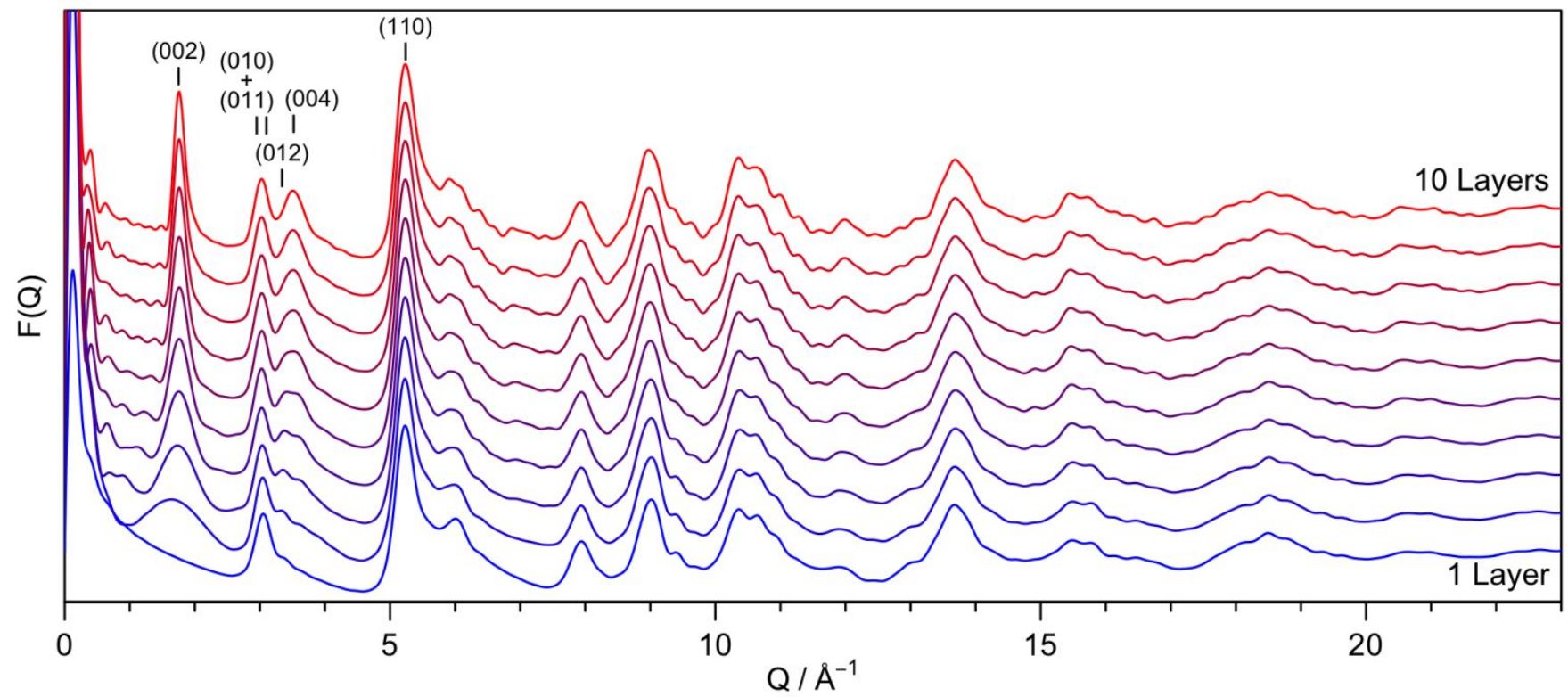

Figure S4: Simulations of the reduced structure function, $F(Q)$, for models with different numbers of identical carbon layers. Peaks below $6 \AA^{-1}$ are indexed based on the graphite unit cell. 


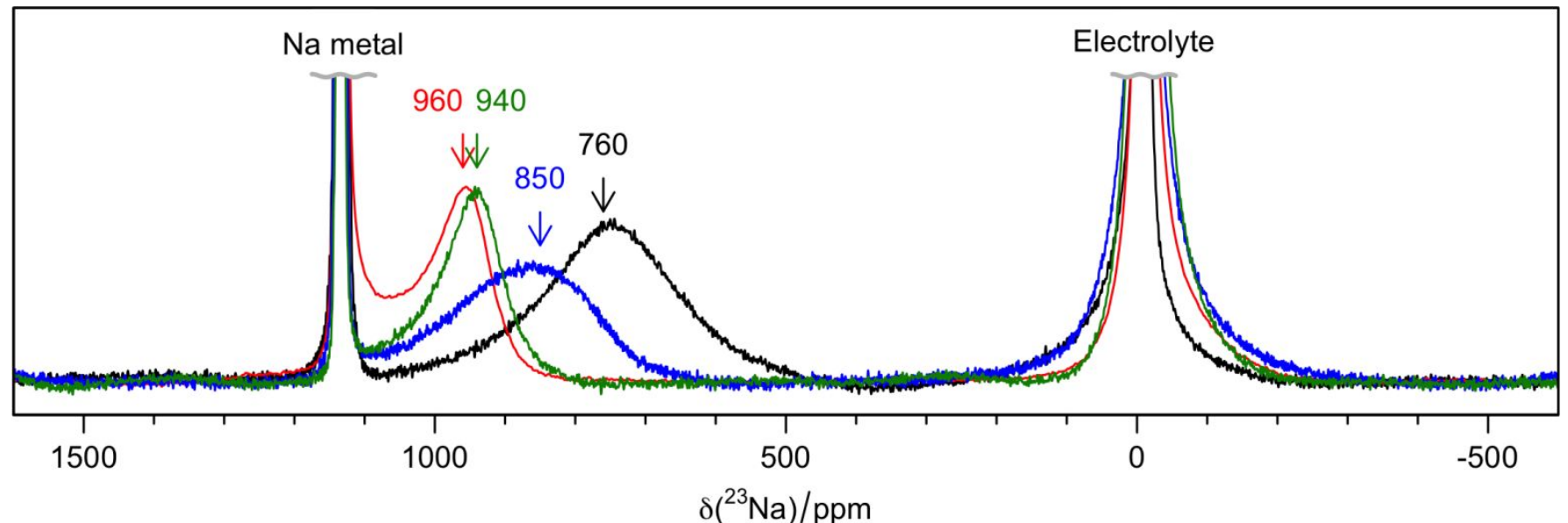

Figure S5: A single spectrum representing the end of discharge from each of the four operando measurements shown in Figure 6. Strong features corresponding largely to the electrolyte or metal have been truncated for clarity, and shifts are indicated for the peaks corresponding to sodium within the hard carbon structures. Black represents Carbon A, red Carbon B, blue $1100^{\circ} \mathrm{C}$, and green $1500^{\circ} \mathrm{C}$. 


\section{TOTAL SCATTERING DATA FOR CARBON WITH ELECTROLYTE}

For all ex situ total scattering measurements, the data obtained are that of the pure carbon. For operando measurements, however, it is unavoidable to also include the electrolyte in the data. In order to asses the effect of including the electrolyte in the measurement, two samples were prepared:

1. A sample of the pristine electrode material containing carbon A and $10 \% \mathrm{pVDF}$ binder.

2. An identical sample to 1 , which was flooded with $1 \mathrm{M} \mathrm{NaPF}_{6}$ in propylene carbonate.

Both samples were placed in cell bodies identical to those used in the operando PDF measurements and measured on beamline I15-1 at Diamond Light Source in the same manner as the other ex situ measurements. Results are shown in Figure S6, where a difference curve is also shown to highlight the contribution of the electrolyte. It is important to note that in our operando measurements the contribution of the electrolyte should remain effectively constant as a function of time; as we have employed a differential analysis of these data, which only considers changes between samples, correlations resulting from the electrolyte should not be present in any of the differential PDFs and therefore will not affect the analysis.
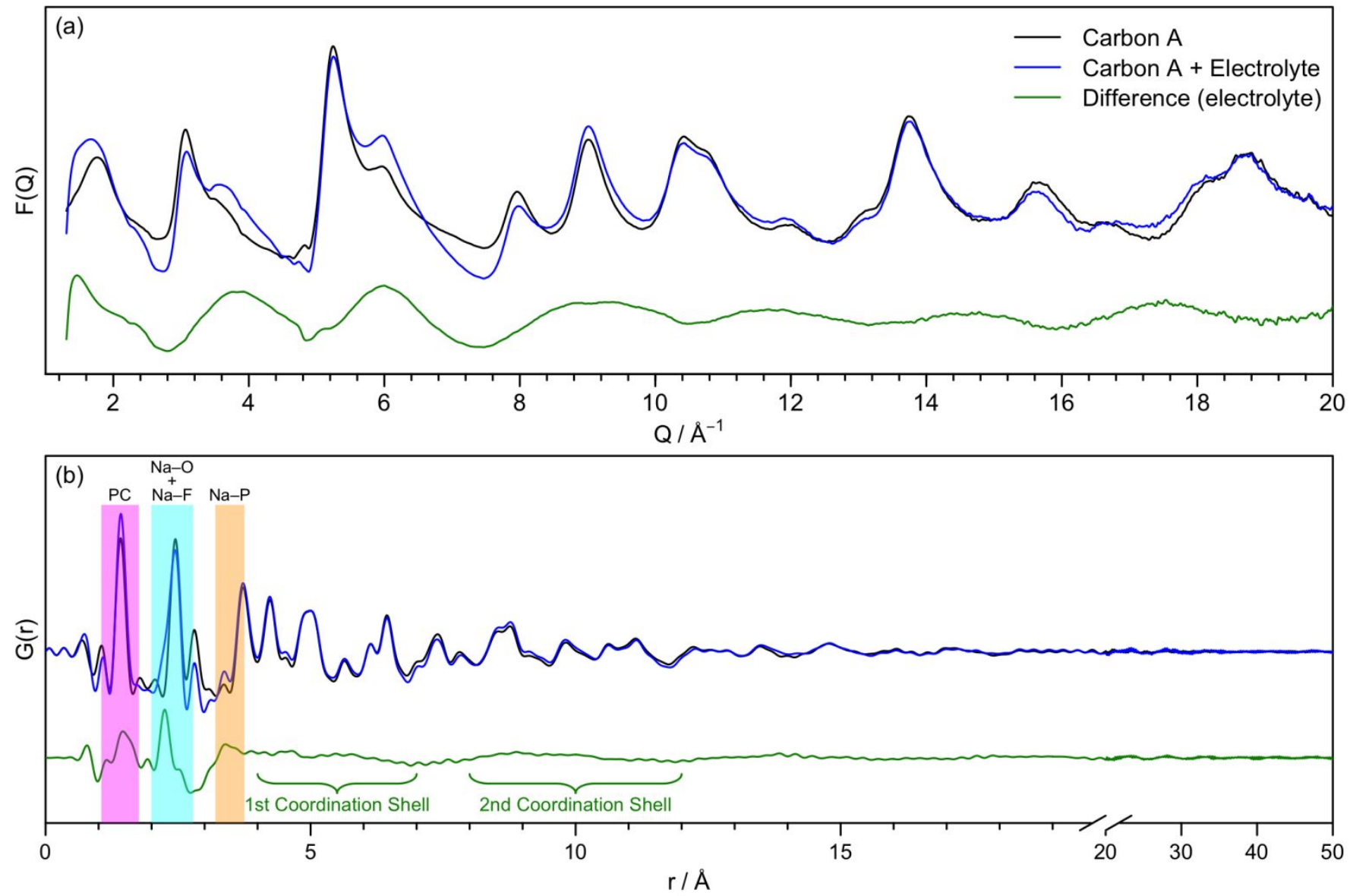

Figure S6: (a) The reduced structure function, $F(Q)$, measured for Carbon A (black) and the same carbon soaked in electrolyte $-\mathrm{NaPF}_{6}$ in $\mathrm{PC}$ (blue). The difference (representing the electrolyte) is offset below in green. (b) The extracted PDFs corresponding to the F(Q)s in (a). Regions of the difference curve (i.e. the electrolyte) are highlighted based on their likely origin: correlations within a molecule of propylene carbonate $^{4}$ are highlighted in pink, Na-O and Na-F correlations in blue and Na-P in orange; ${ }^{5}$ broad regions corresponding to coordination shells are also indicated. 


\section{SMALL ANGLE SCATTERING DATA FOR CARBON B}

Small Angle X-ray Scattering (SAXS) measurements were made on a Xenocs Xeuss 2.0 equipped with a $\mathrm{Cu} \mathrm{ka}$ source collimated by two sets of scatterless slits. A Pilatus 300k detector mounted on a translation stage was used to record the scattered signal. Measurements were made with multiple samples to detector distances with the results provided here measured at $0.339(2) \mathrm{m}$.

Samples were mounted in a metal washer $0.8 \mathrm{~mm}$ thick with sticky Kapton windows and measured for $10 \mathrm{~min}$. Masks were applied to the collected 2D images which were then radially integrated as a function of $q$ to produce 1D intensity versus $q$ plots. These were then corrected for transmission and background scattering.

The fully corrected SAXS data from three carbon samples, pyrolysed at three different temperature ranges are plotted in Figure S7. Carbon B corresponds to the sample pyrolyzed at $1200-1500{ }^{\circ} \mathrm{C}$.

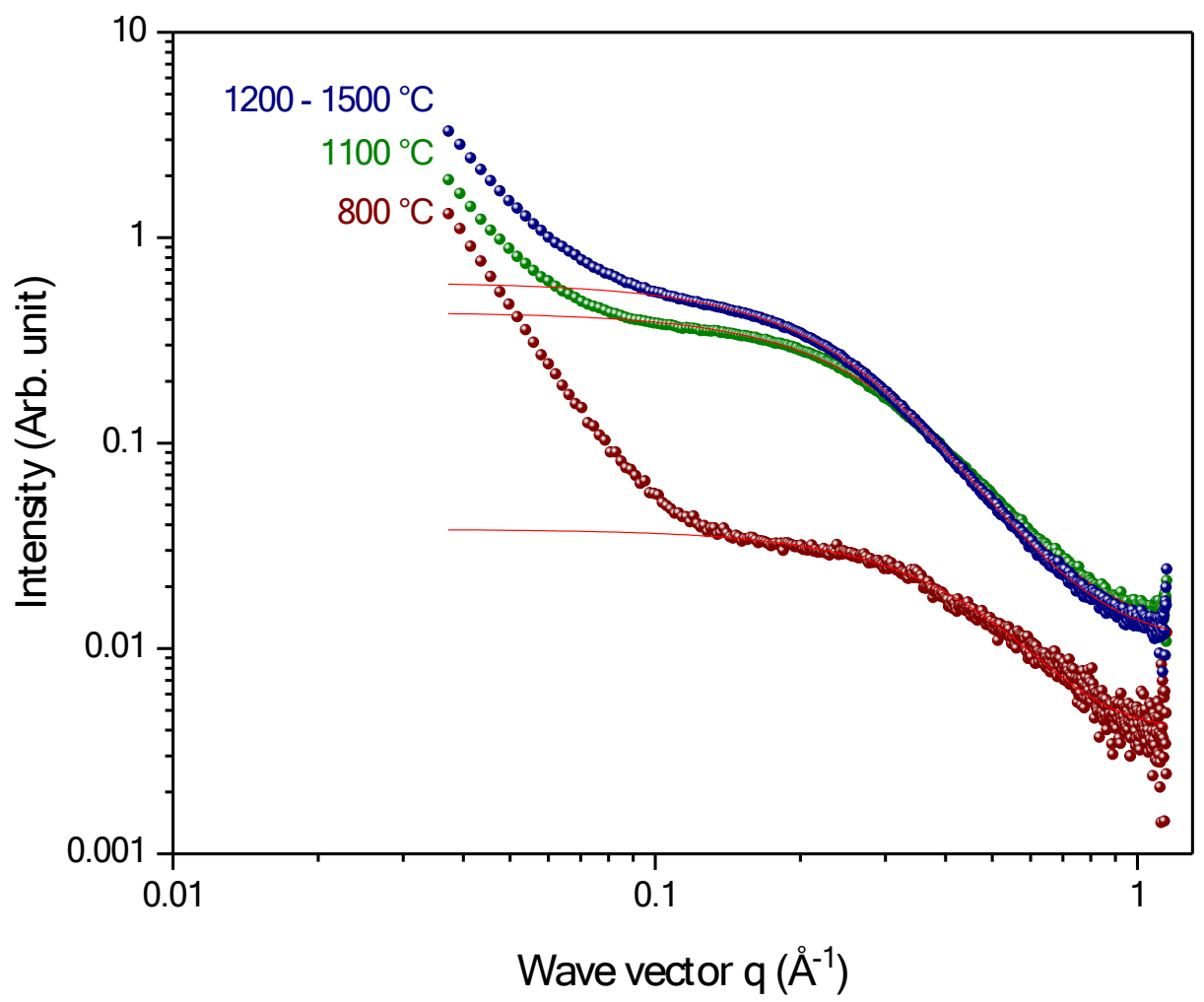

Figure S7: Fully corrected SAXS data from carbon samples pyrolysed at $800{ }^{\circ} \mathrm{C}, 1100{ }^{\circ} \mathrm{C}$ and $1200-$ $1500{ }^{\circ} \mathrm{C}$.

The SAXS data has been modelled using the Unified Fit approach within the Irena analysis package. ${ }^{6,7}$ This allows a combined Guinier and power-law fit to the measured data. The Guinier regime models the curve in the data to give the radius of gyration $(R g)$ and the power-law slope provides information on the surface 
roughness of the pores. $R g$ is the root mean square (RMS) distance to the centre of density of a particle. If the particle is a sphere $R g$ is related to the radius by $R g^{2}=3 / 5 R^{2}$.

Modelling these particles has been made with the assumption that the pore surface is smooth which results in power-law slope of $q^{4}$. In this work the measured scattering signal between 0.1 and $1 \AA^{-1}$ is assumed to be from pores and the scattering from the larger carbon particles produces the power-law slope measured at low $q$ values.

Derived estimated radius and volume of spherical pores as a function of pyrolysis temperature are plotted in Figure S8.

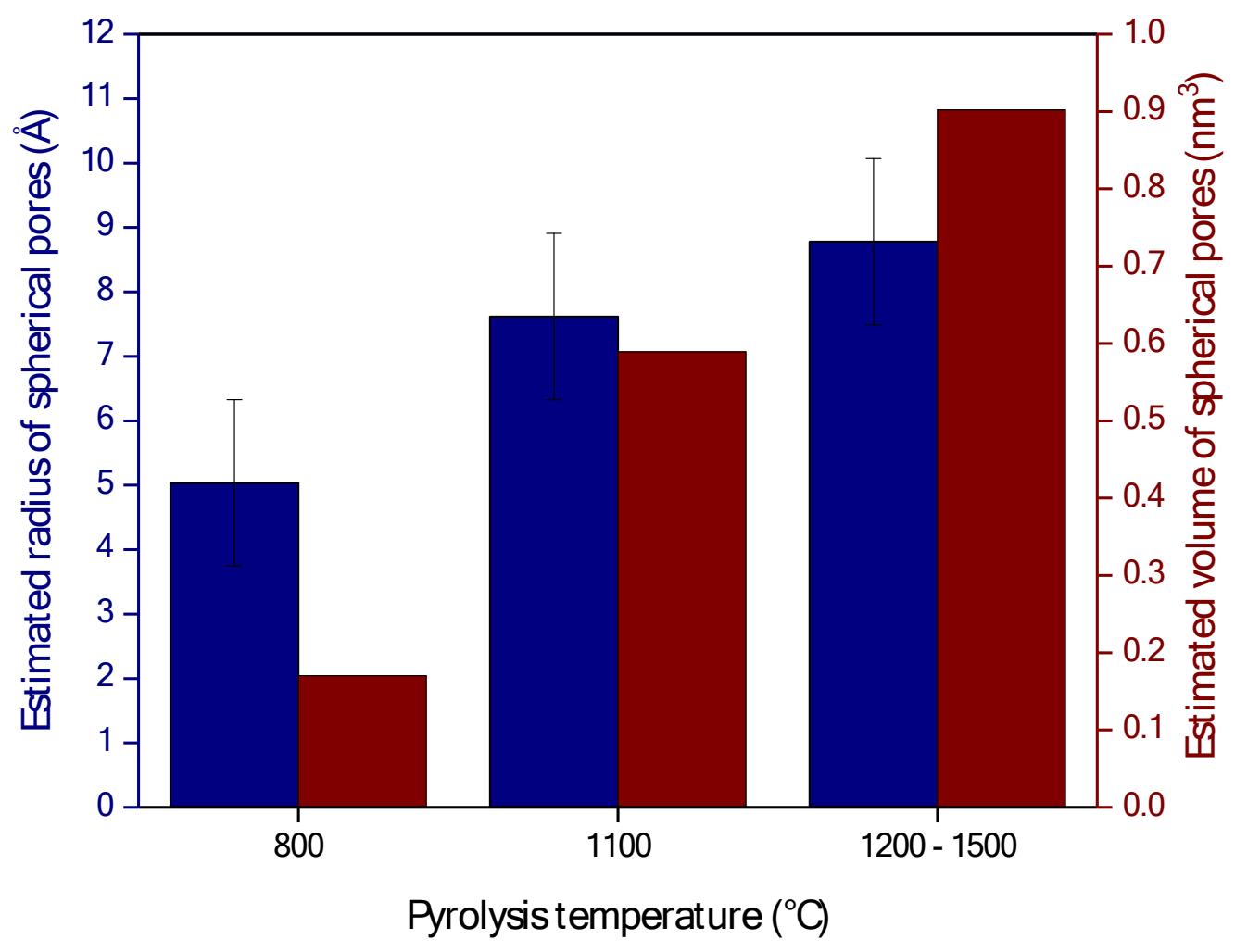

Figure S8: Estimated radius and volume of spherical pores of carbon samples pyrolysed at $800{ }^{\circ} \mathrm{C}, 1100{ }^{\circ} \mathrm{C}$ and $1200-1500{ }^{\circ} \mathrm{C}$.

At pyrolysis temperatures below $1500{ }^{\circ} \mathrm{C}$, the average internal pore diameter is estimated to be in the range of 1-2 nm. Although, the charring step is reported to be an influential parameter, ${ }^{8}$ employing higher pyrolysis temperatures evidently leads to expansion of internal pores. This observation is consistent with earlier reports in literature. ${ }^{9}$ 


\section{DQ/DV DATA FOR EACH CARBON}

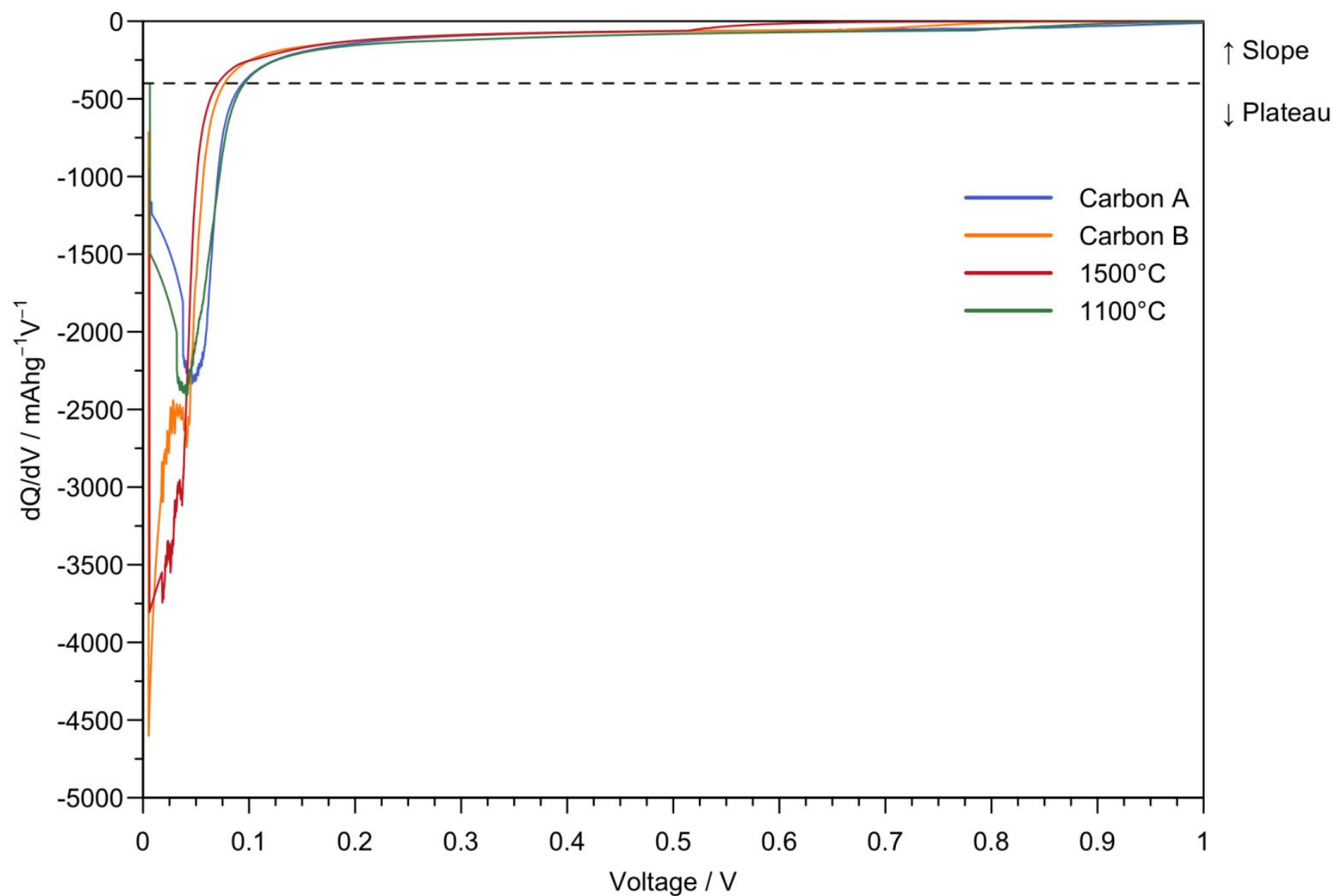

Figure S9: dQ/dV data for sodium-hard carbon cells cycled at a rate of $\mathrm{C} / 20$ (corresponding to achieving a capacity of $300 \mathrm{mAhg}^{-1}$ in 20 hours) between 2 and $0.005 \mathrm{~V}$ for Carbon A (blue), Carbon B (orange), $1500{ }^{\circ} \mathrm{C}$ (red), and $1100{ }^{\circ} \mathrm{C}$ (green). The cutoff value at which the plateau process begins is indicated with a dashed line at $d Q / d V=-400 \mathrm{mAhg}^{-1} \mathrm{~V}^{-1}$. A plot of voltage vs capacity for these data can be found in Figure 3. 


\section{SCALE FACTORS FOR OPERANDO PDF}

For the operando measurements, a number of issues outside of our control mean that the intensity of the raw data is not comparable between different scans on the same sample:

- The beam position of the beamline is not controlled within a feedback loop, which results in an inconsistent photon flux as a function of time; with no I0 monitor to allow for the correction of this post-measurement

- The volume expansion of the electrode results in a changing volume (and possibly density) of material within the beam

- It is not possible to know the exact composition of the section of the electrode we are observing

As such it was necessary to rescale the data after collection. PDFs were rescaled such that the total intensity over the range $15 \AA<r<25 \AA$ remained constant as a function of time. This range was chosen to be greater than the range over which we observe changes in the PDFs. The region over which data are rescaled has an effect on the intensity of the first two peaks in the PDFs (and thus in the differential PDFs calculated from them), but no significant impact beyond this.
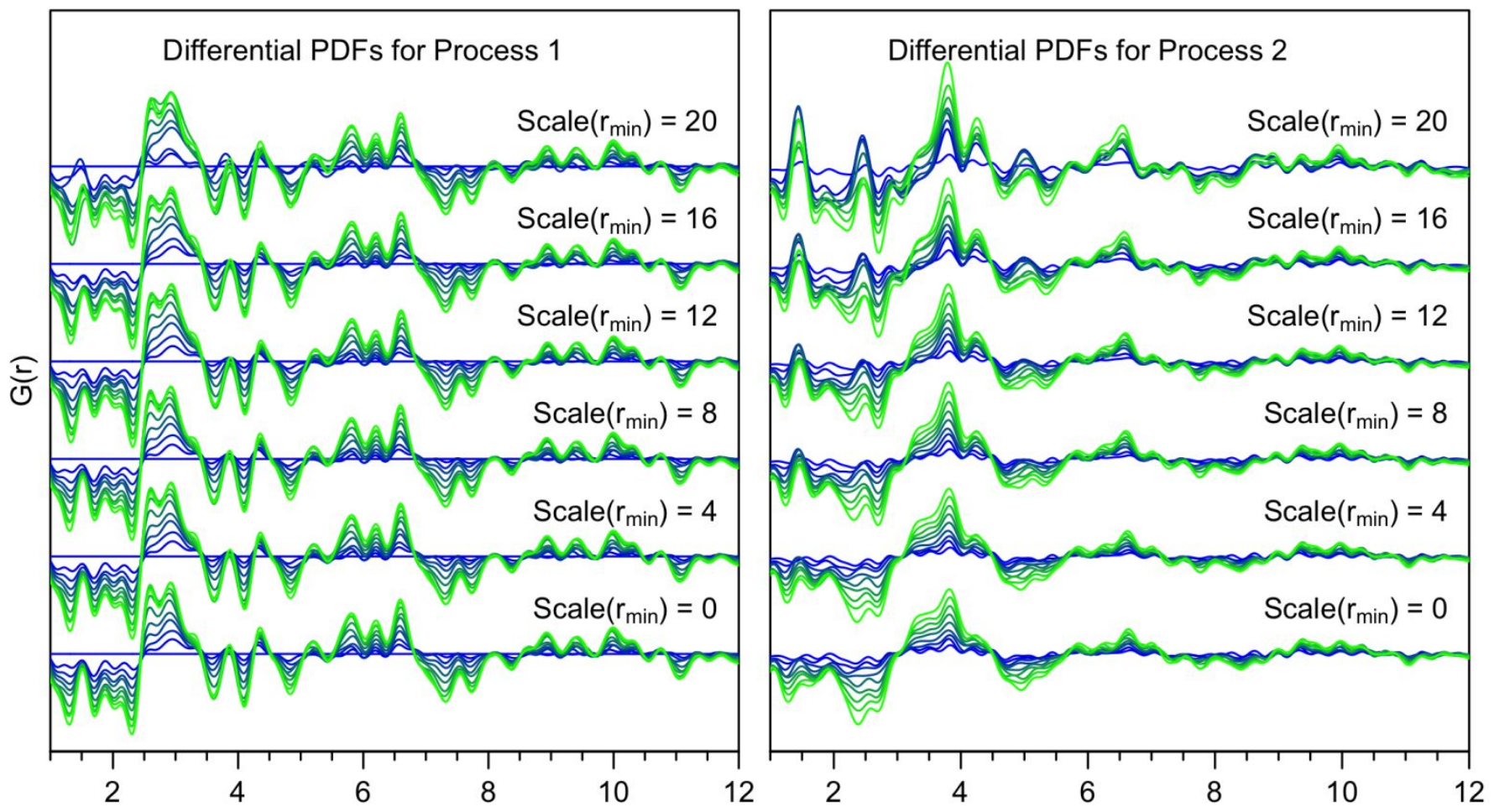

Figure S10: The effect of different lower bounds for the region over which data are rescaled, i.e. $r_{\min }<r<$ $25 \AA$. The left hand plot corresponds to the first electrochemical process (the sloping region), the right to the second (the plateau); in each case differential PDFs are plotted with blue lines corresponding to the start of the process and green to the end. 


\section{DIFFERENTIAL PDFS DURING THE CHARGE PROCESSES}

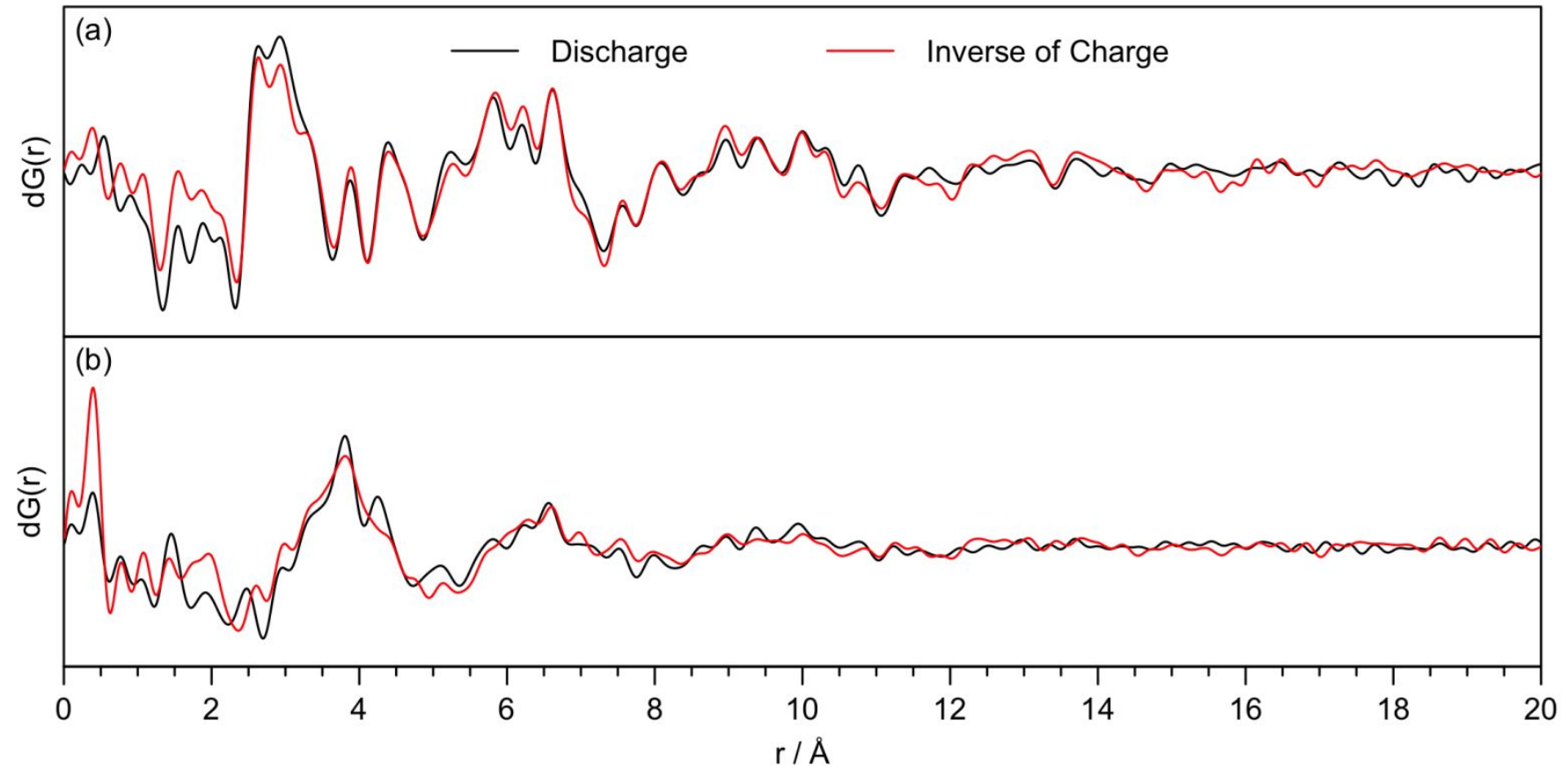

Figure S11: Comparison of the differential PDFs obtained at the end of the (a) slope and (b) plateau processes on discharge (black) and charge (red). The dPDFs for the charge processes have been inverted (multiplied by -1) for easier comparison with the discharge dPDFs. 


\section{CREATING A CURVED GRAPHENE FRAGMENT}

PDFs for the pristine carbons were modelled as curved graphene fragments. Curvature being introduced by constraining a graphene layer to lie on the surface of an imaginary ellipsoid. The radii of curvature of this ellipsoid may then be changed to adjust the curvature in the model. These fragments are created from a planar, rectangular graphene fragment of width $x$ and height $y$ by placing it at the centre of a box with dimensions $1 \times 1 \times 1$ such that each atom $(i)$ has normalised coordinates $\left(x_{\text {layer }, i}, y_{\text {layer }, i}, 0\right)$. An ellipsoid with radii $\left(A^{-0.5}, B^{-0.5}, 0.5\right)$ is placed at $(0.5,0.5,0.5)$ such that it just touches the graphene layer at its lowest point. The ellipsoid is described by the following expression:

$$
A\left(x_{\text {ellipsoid }}-0.5\right)^{2}+B\left(y_{\text {ellipsoid }}-0.5\right)^{2}+4\left(z_{\text {ellipsoid }}-0.5\right)^{2}=1
$$

We create a model where the carbon atoms are placed at the intersection of a line drawn from each atom to the centre of the ellipsoid with the surface of the ellipsoid, the parametric equations for these lines are as follows:

$$
\begin{gathered}
x_{\text {line }, i}=x_{\text {layer }, i}+t\left(0.5-x_{\text {layer }, i}\right) \\
y_{\text {line }, i}=y_{\text {layer }, i}+t\left(0.5-y_{\text {layer }, i}\right) \\
z_{\text {line }, i}=0.5 t+n d
\end{gathered}
$$

Where the parameter $n$ is included to allow us to create multiple identical layers separated by a distance $d$, it takes integer values from 0 to one fewer than the total number of layers. The points of intersection are then given by solving the following equation:

$$
\begin{gathered}
\left\{A\left(0.5-x_{\text {layer }, i}\right)^{2}+B\left(0.5-y_{\text {layer }, i}\right)^{2}+1\right\} t_{i}^{2} \\
+2\left\{A x_{\text {layer }, i}^{2}+B y_{\text {layer }, i}-B y_{\text {layer }, i}^{2}-0.25 A-0.25 B-1\right\} t_{i} \\
+A x_{\text {layer }, i}^{2}-A x_{\text {layer }, i}+B y_{\text {layer }, i}^{2}-B y_{\text {layer }, i}+0.25 A+0.25 B=0
\end{gathered}
$$

Solving for $t_{i}$ gives the coordinates for atom $i$ on the curved surface of the ellipsoid:

$$
t_{i}=\frac{-b-\sqrt{b^{2}-4 a c}}{2 a}
$$

Where:

$$
\begin{aligned}
& a_{i}=A\left(0.5-x_{\text {layer }, i}\right)^{2}+B\left(0.5-y_{\text {layer }, i}\right)^{2}+1 \\
& b_{i}=2\left(A\left(x_{\text {layer }, i}-x_{\text {layer }, i}^{2}\right)+B\left(y_{\text {layer }, i}-y_{\text {layer }, i}^{2}\right)-0.25 A-0.25 B-1\right) \\
& c_{i}=A\left(x_{\text {layer }, i}^{2}-x_{\text {layer }, i}\right)+B\left(y_{\text {layer }, i}^{2}-y_{\text {layer }, i}\right)+0.25 A+0.25 B
\end{aligned}
$$

In addition, the coordinates in each direction are scaled by a factor in the same manner as the lattice parameters $a, b$, and $c$ for a crystal. As the initial model is $2 \mathrm{D}$, curvature around the $c$ axis would only affect the in plane bonding, and is therefore not considered. The parameters $A, B$, and $d$ may be refined along with the scale factors. 


\section{DIFFERENTIAL PDF DECONVOLUTION METHOD}

We created a PDF for an expanded carbon matrix by taking the PDF acquired before sodium insertion, and expanding the $r$-axis in a linear manner $\left(r_{\text {expanded }}\right)$. To assess whether this is a valid model for the sharp component of the differential, we need to convert this into a differential PDF by subtracting from it the PDF for the pristine material $\left(d G_{\text {sharp }}(r)\right)$. The broad component $\left(d G_{\text {broad }}(r)\right)$ is then simply the difference between the measured differential PDF and this calculated differential.

$$
\begin{gathered}
d G(r)=G_{\text {end of slope }}(r) / s_{1}-G_{\text {pristine }}(r) \\
r_{\text {expanded }}=r_{\text {add }}+r_{\text {min }}+\left(r-r_{\text {min }}\right) r_{\text {multi }} \\
d G_{\text {sharp }}(r)=s_{2}\left(G\left(r_{\text {expanded }}\right)-G(r)\right) \\
d G_{\text {broad }}(r)=d G(r)-d G_{\text {sharp }}(r)
\end{gathered}
$$

Where $s_{1}$, and $s_{2}$ represent scale factors, $r_{\text {add }}$ represents the additive expansion of the carbon matrix, $r_{\text {multi }}$ represents the multiplicative expansion of the carbon matrix, and $r_{\min }$ represents the distance below which expansion does not occur. The necessity of scale factors is discussed earlier.

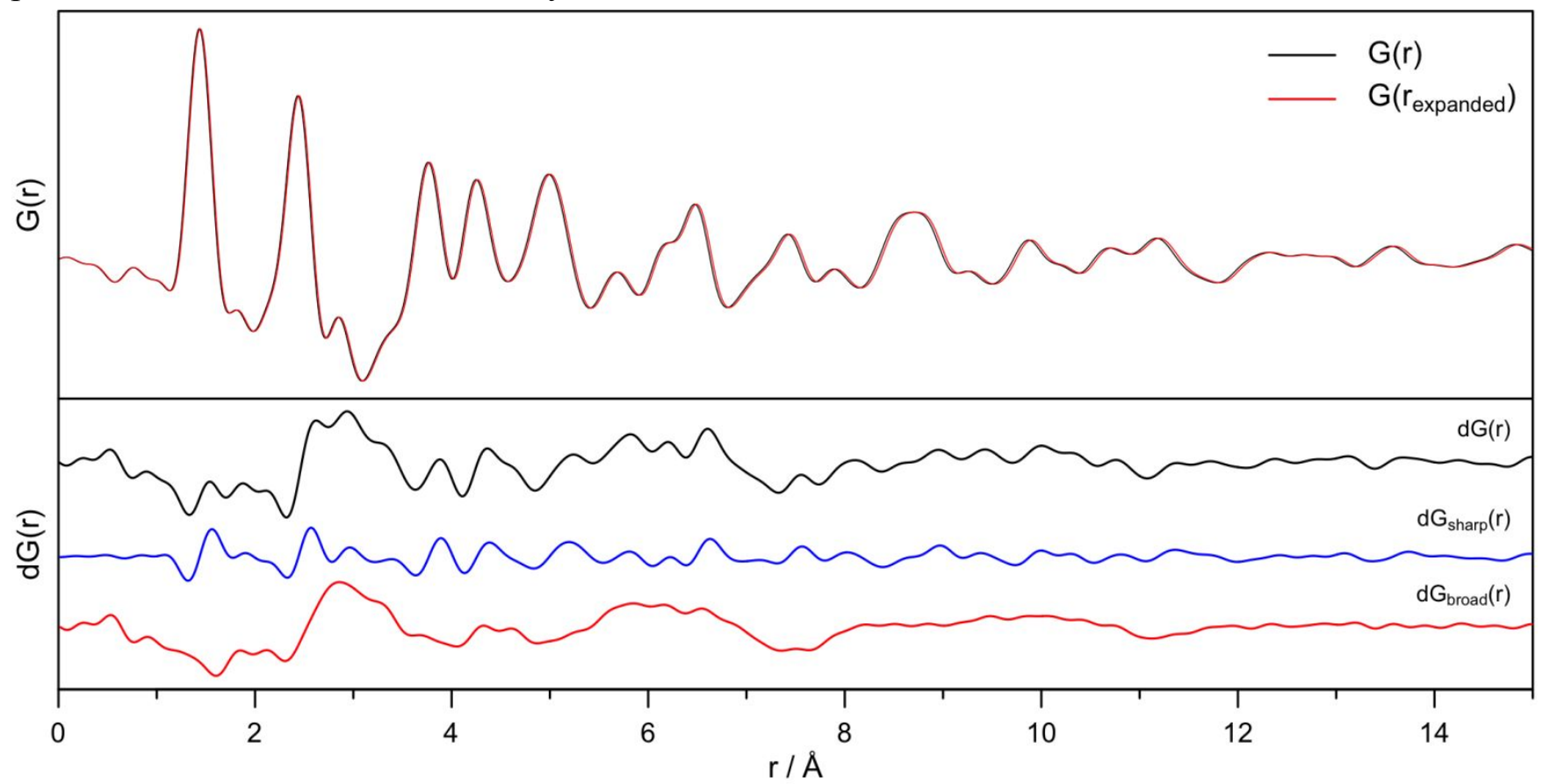

Figure S12: (top) The PDFs for Carbon A measured before sodium insertion (black) and the same measurement with a simulated expansion to the $r$-axis (red). (bottom) The differential PDF corresponding to the end of the first (sloping) electrochemical process (black) and its deconvolution into sharp (blue) and broad (red) components.

If expanded carbon is a good model for the sharp component, we will be able to find values for $r_{\text {add }}, r_{\text {multi }}$, and $r_{\min }$ that give a smooth broad component. We optimised these parameters by minimising the sum of squares of the first derivative of the broad component:

$$
\sum_{r}\left\{\frac{\partial G_{\text {broad }}\left(r, s_{1}, s_{2}, r_{\text {add }}, r_{\text {min }}, r_{\text {multi }}\right)}{\partial r}\right\}^{2}
$$

The final deconvolution is shown in Figure S12 above and in Figure 7a of the main text, and is shown for intermediate steps between the start of discharge and the end of process 1 in Figure S13. It is clear that this deconvolution method is able to effectively separate a sharp component from the underlying broad component. This demonstrates that a combination of expansion of the carbon matrix along with the growth of a more disordered phase is a valid model for the structural changes during this region of the electrochemistry. 


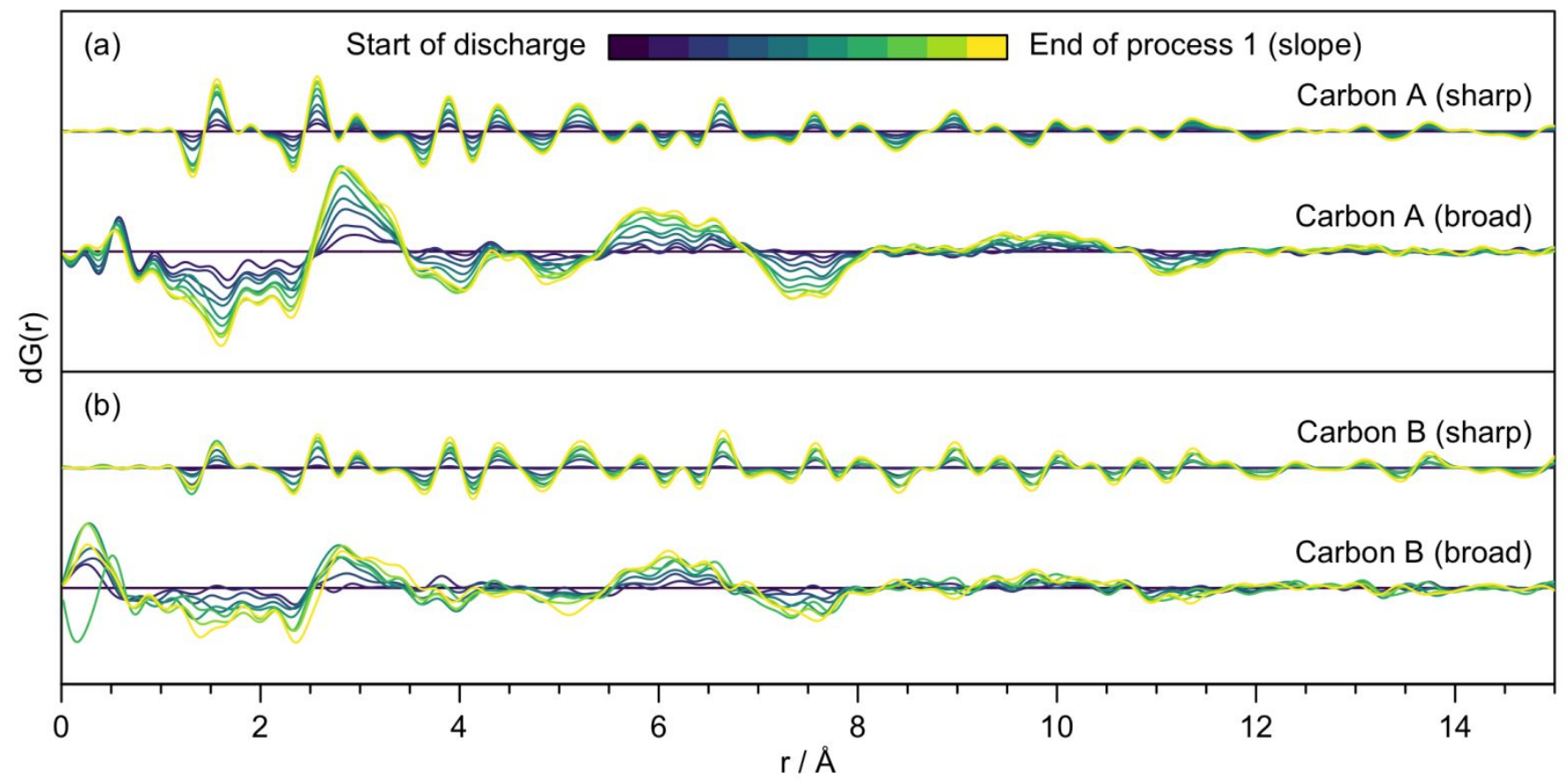

Figure S13: The evolution of the sharp and broad components of the deconvolution described above for both carbons A and B during the first electrochemical process. 


\section{MODELLING THE BROAD COMPONENT OF THE DIFFERENTIAL PDF FOR THE SLOPING PROCESS}

In order to understand the changes to the PDF observed during the sloping process, we first considered the contribution to the PDF resulting from the addition of sodium atoms near to the carbon layers. A curved graphene fragment, sized $18 \times 18 \AA$, was created using the DiffPy-CMI complex modelling infrastructure software. ${ }^{3}$ A single sodium atom was placed a variable distance above the centre of the layer (and directly above the centre of a 6-membered ring). Partial PDFs were calculated for a variety of distances, where only $\mathrm{Na}-\mathrm{C}$ correlations were included in the calculations. The results are shown in Figure S14.

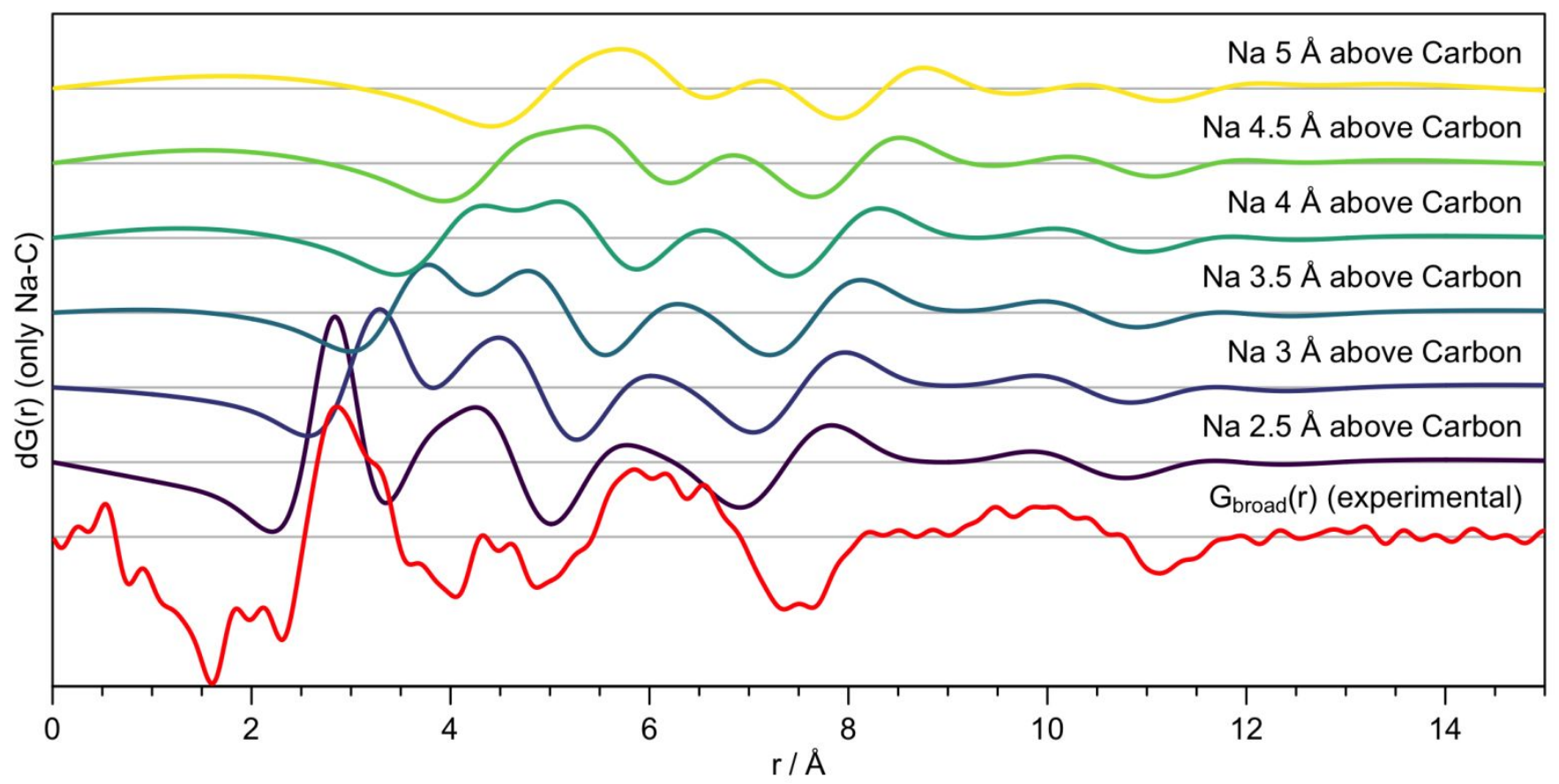

Figure S14: Change in the PDF for a single hard carbon layer with a single sodium atom placed above at distances between 2.5 and $5 \AA$.

We then performed a similar simulation with two layers, the sodium atom being centred between them. Results are shown in Figure S15.

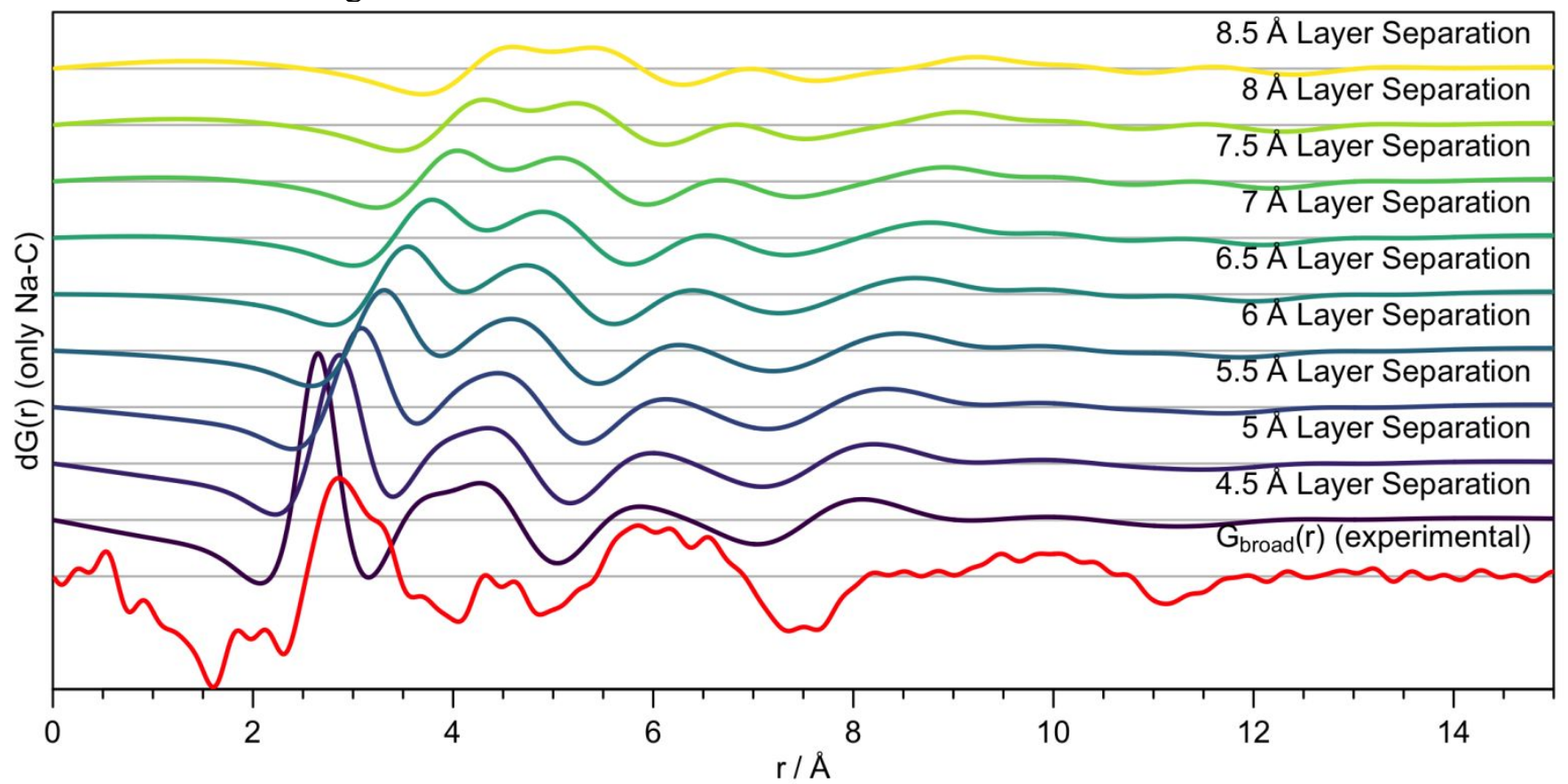

Figure S15: Change in the PDF for two hard carbon layers with a single sodium atom centred between them. The layer separation was varied between 4.5 and $8.5 \AA$. 
It is clear that similar correlations to those observed in the experimental differential PDF can be generated from these simple models, providing the distance between the sodium atom and the carbon layers is set appropriately. We therefore considered a range of simple structures to assess the similarity of their simulated dPDFs and the experimental data. The structures are shown in Figure S16, and their calculated partial PDFs (computed with the same method as above) are presented in Figure S17.

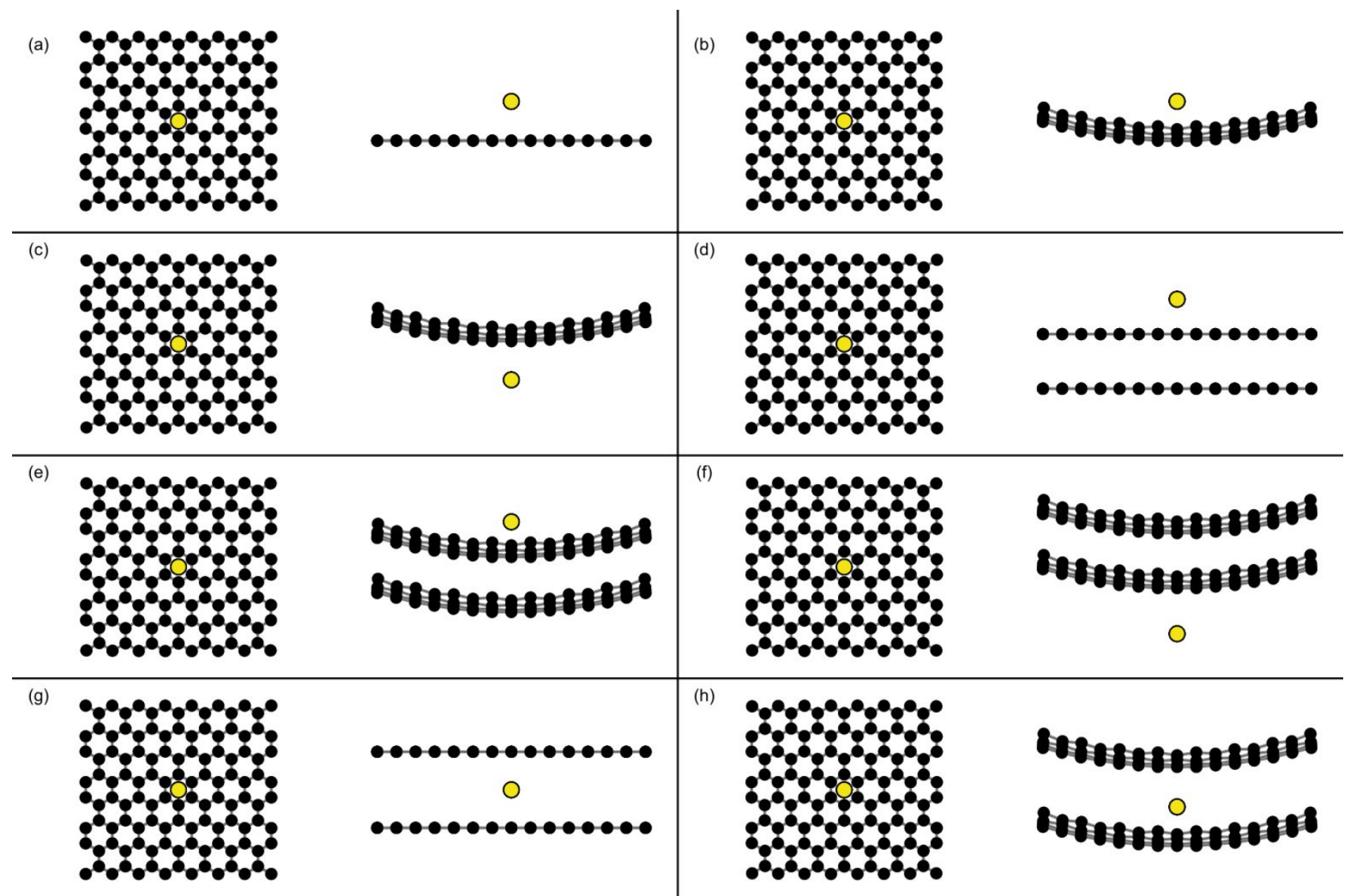

Figure S16: Structures used for the PDF simulations in Figures S17 and S19. 


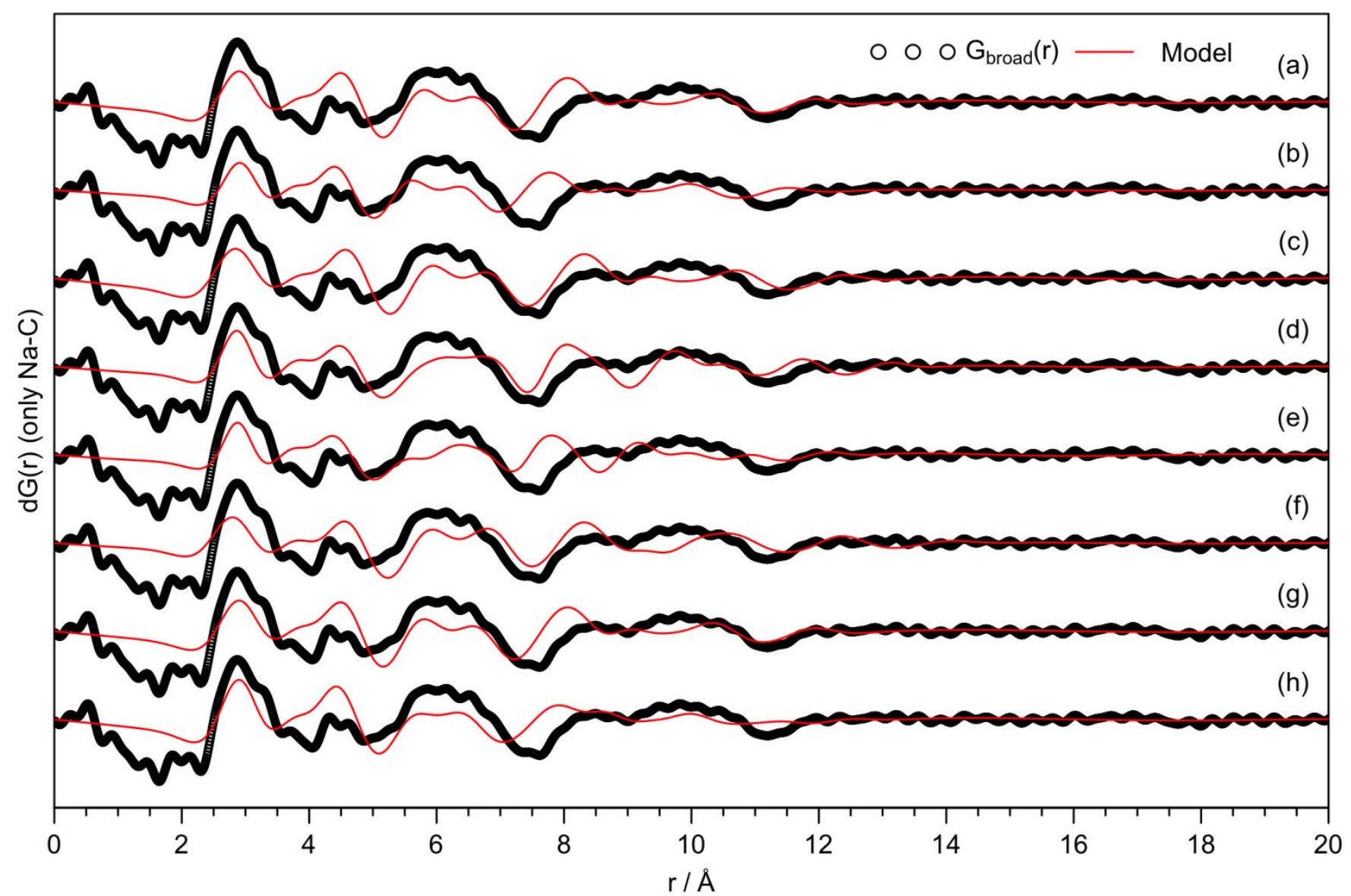

Figure S17: Simulated differential PDFs for the structures shown in Figure S16. Only Na-C correlations were included in the calculations.

Whilst the above models provide a reasonable match to the experimental data, they do not account for any changes to the carbon structure beyond the expansion of the $\mathrm{C}-\mathrm{C}$ bond length (see discussion relating to the deconvolution of sharp and broad components). In particular, any expansion to the interlayer distance, or disordering of the layers, is not considered. In order to assess the importance of any changes to this distance, we created models containing two curved graphene layers with the interlayer distance being varied between 3.7 and 5 Å. A PDF was simulated for each configuration, and the results are shown in Figure S18. 

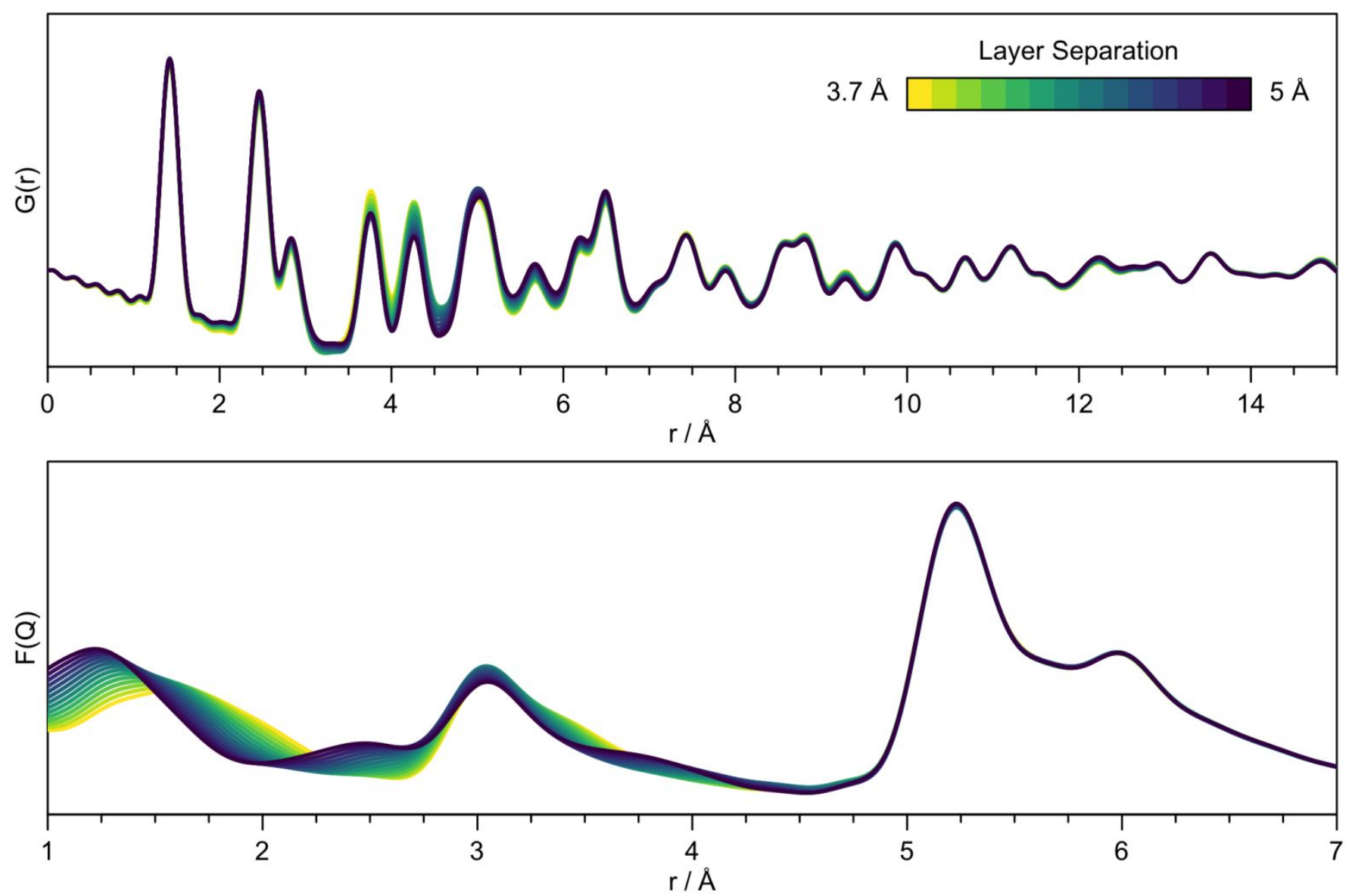

Figure S18: Change in the PDF for simulated hard carbons with layer separations between 3.7 and $5 \AA$.

We can adjust our simulations to account for this change by including both $\mathrm{Na}-\mathrm{C}$ and $\mathrm{C}-\mathrm{C}$ correlations in our calculation, then subtracting a calculated PDF for the pristine carbon. These models will be sensitive to the ratio of sodium: carbon atoms; in order to retain the simplicity of the models, we simulate a 15-fold increase to the scattering power of sodium. This is approximately the ratio observed electrochemically. By increasing the scattering power rather than the number of atoms, we are explicitly excluding effects resulting from the arrangement of sodium, which may not be appropriate in an average local structure model. The results of these simulations are shown in Figure S19. 


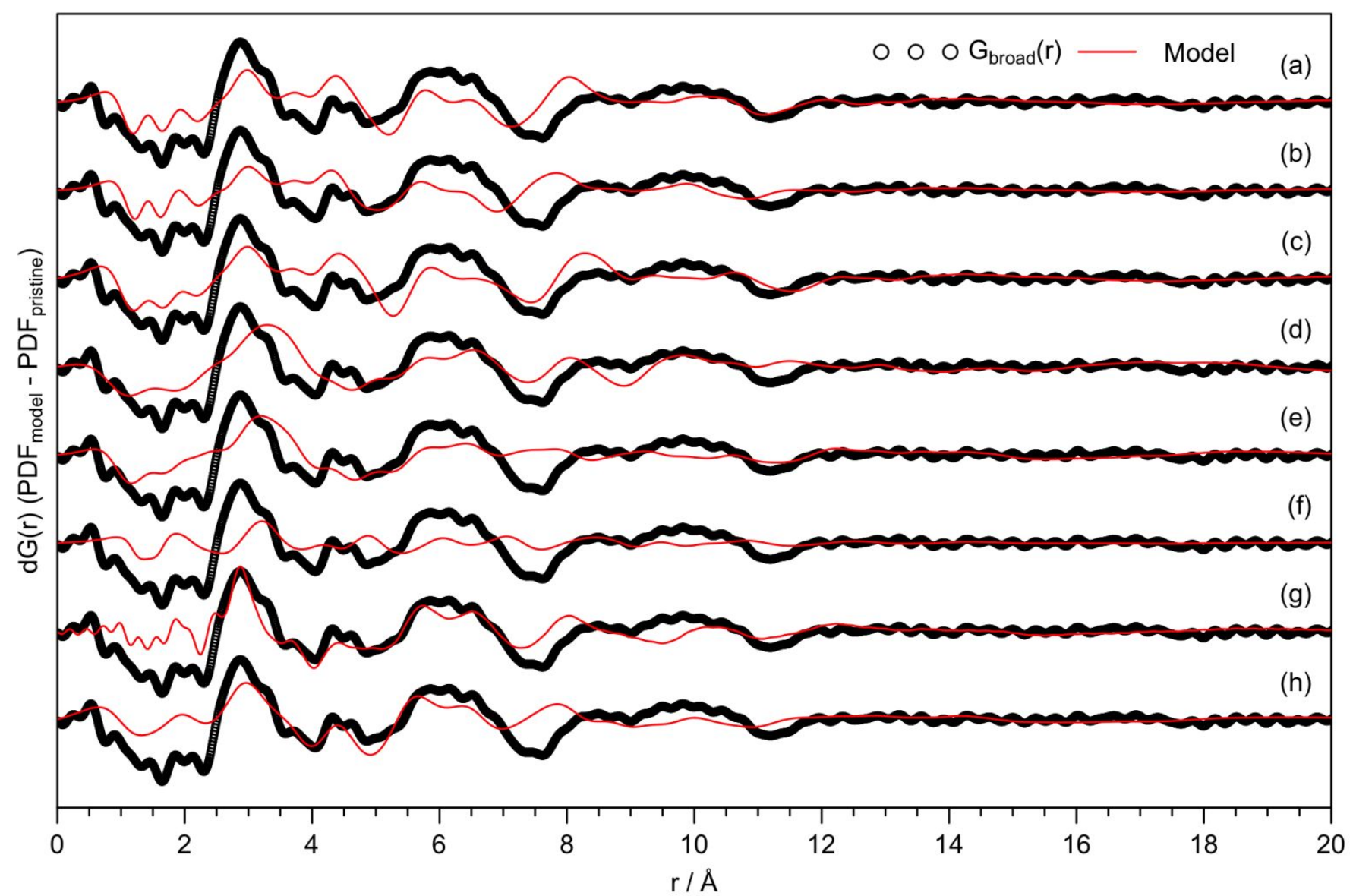

Figure S19: Simulated differential PDFs for the structures shown in Figure S16. Na-C and C-C correlations were included, the simulated PDF for the pristine material was subtracted to generate a differential PDF. The scattering power of sodium was increased by a factor of 15 to simulate the ratio of sodium : carbon expected from the electrochemistry.

It is clear that even when changes to the carbon arrangement are included in the models, there are still a range of structures that adequately explain the experimental data. We therefore suggest that it is best to consider the mechanism for this stage of the electrochemistry to involve the insertion of sodium into a number of different parts of the carbon structure, to result in a number of different carbon-Na arrangements, some of which may be considered intercalation, others pore-coating. 


\section{CALCULATED PDFS FOR DIFFERENT SODIUM CLUSTER DIAMETERS}

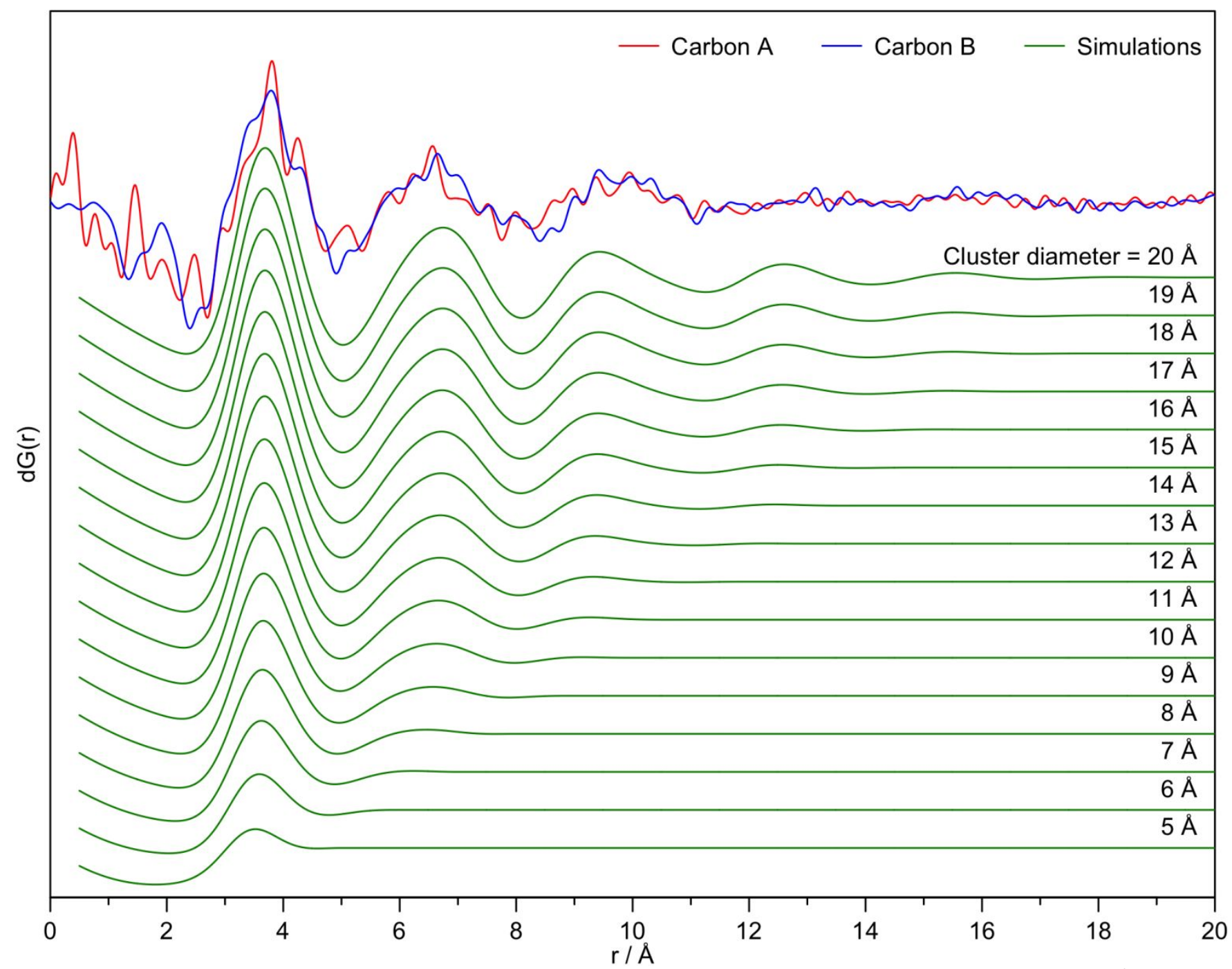

Figure S20: Simulated PDFs for sodium clusters with spherical particle diameters between 5-20 A (green). The experimental differential PDFs for Carbon A (red) and Carbon B (blue) obtained at the end of the plateau process are shown for comparison.

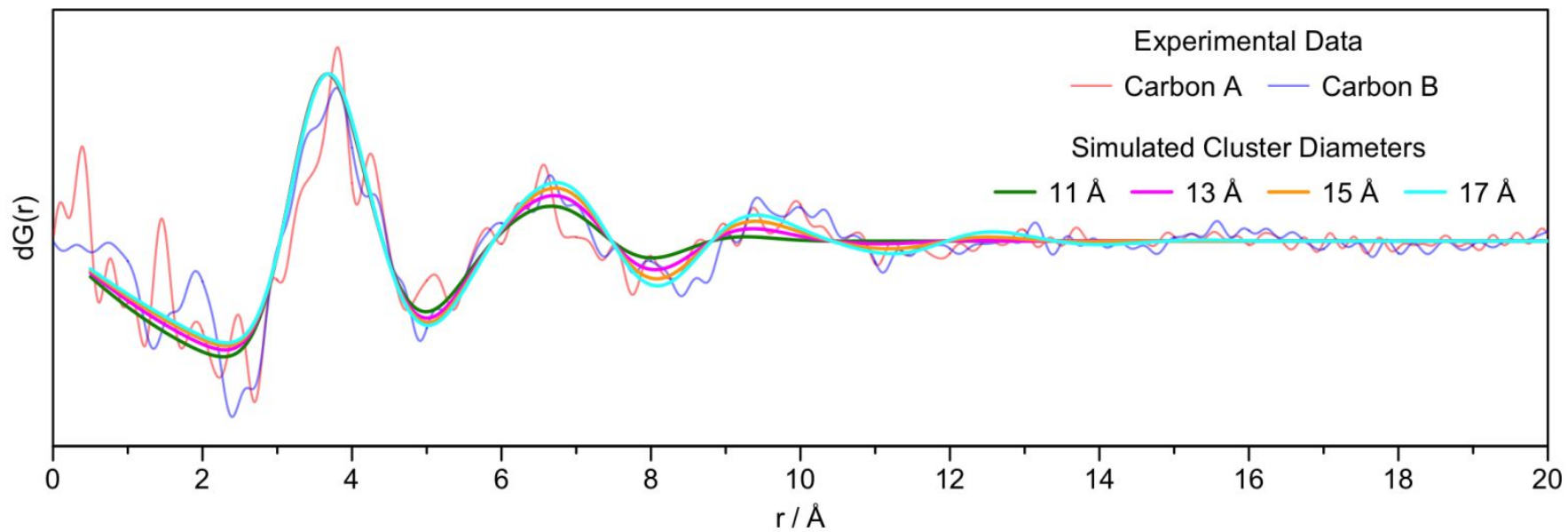

Figure S21: A closeup of Figure S20, including only simulated sodium clusters of $11 \AA$ (green), $13 \AA$ (pink), $15 \AA$ (orange), and $17 \AA$ (cyan), along with the experimental differential PDFs for Carbon A (red) and Carbon B (blue) obtained at the end of the plateau process. Intensities for the simulated clusters have been normalized to give the same intensity for the first peak. This highlights the sensitivity of the method, with small changes in diameter effecting significant changes in correlation length and peak intensity notably at $11 \AA$ diameter, the broad correlation around $8-11 \AA$ is missing, whereas at $17 \AA$ diameter additional correlations would be expected beyond $11 \AA$, which are not discernable in the experimental data. 


\section{REFERENCES}

(1) Basham, M.; Filik, J.; Wharmby, M. T.; Chang, P. C. Y.; El Kassaby, B.; Gerring, M.; Aishima, J.; Levik, K.; Pulford, B. C. A.; Sikharulidze, I.; Sneddon, D.; Webber, M.; Dhesi, S. S.; Maccherozzi, F.; Svensson, O.; Brockhauser, S.; Náray, G.; Ashton, A. W. Data Analysis WorkbeNch (DAWN). J. Synchrotron Radiat. 2015, 22, 853-858. https://doi.org/10.1107/S1600577515002283.

(2) Qiu, X.; Thompson, J. W.; Billinge, S. J. L. PDFgetX2 : A GUI-Driven Program to Obtain the Pair Distribution Function from X-Ray Powder Diffraction Data. J. Appl. Crystallogr. 2004, 37 (4), 678. https://doi.org/10.1107/S0021889804011744.

(3) Juhás, P.; Farrow, C. L.; Yang, X.; Knox, K. R.; Billinge, S. J. L. Complex Modeling: A Strategy and Software Program for Combining Multiple Information Sources to Solve Ill Posed Structure and Nanostructure Inverse Problems. Acta Crystallogr. Sect. A Found. Adv. 2015, 71, 562-568. https://doi.org/10.1107/S2053273315014473.

(4) Delavoux, Y. M.; Gilmore, M.; Atkins, M. P.; Swadźba-Kwaśny, M.; Holbrey, J. D. Intermolecular Structure and Hydrogen-Bonding in Liquid 1,2-Propylene Carbonate and 1,2-Glycerol Carbonate Determined by Neutron Scattering. Phys. Chem. Chem. Phys. 2017, 19 (4), 2867-2876. https://doi.org/10.1039/c6cp07790k.

(5) Jensen, A. C. S.; Au, H.; Gärtner, S.; Titirici, M.; Drew, A. J. Solvation of NaPF 6 in Diglyme Solution for Battery Electrolytes. Batter. Supercaps 2020, 3 (12), 1306-1310. https://doi.org/10.1002/batt.202000144.

(6) Beaucage, G. Approximations Leading to a Unified Exponential/Power-Law Approach to SmallAngle Scattering. J. Appl. Crystallogr. 1995, 28 (6), 717-728. https://doi.org/10.1107/s0021889895005292.

(7) Ilavsky, J.; Jemian, P. R. Irena: Tool Suite for Modeling and Analysis of Small-Angle Scattering. $J$. Appl. Crystallogr. 2009, 42 (2), 347-353. https://doi.org/10.1107/S0021889809002222.

(8) Yamamoto, H.; Muratsubaki, S.; Kubota, K.; Fukunishi, M.; Watanabe, H.; Kim, J.; Komaba, S. Synthesizing Higher-Capacity Hard-Carbons from Cellulose for Na- and K-Ion Batteries. J. Mater. Chem. A 2018, 6 (35), 16844-16848. https://doi.org/10.1039/c8ta05203d.

(9) Kamiyama, A.; Kubota, K.; Nakano, T.; Fujimura, S.; Shiraishi, S.; Tsukada, H.; Komaba, S. HighCapacity Hard Carbon Synthesized from Macroporous Phenolic Resin for Sodium-Ion and Potassium-Ion Battery. ACS Appl. Energy Mater. 2020, 3 (1), 135-140. https://doi.org/10.1021/acsaem.9b01972. 